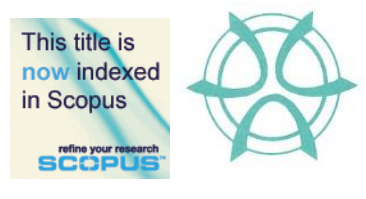

PLANNING MALAYSIA:

Journal of the Malaysian Institute of Planners

VOLUME XIV (2016), Page 29 - 38

\title{
SUSTAINABLE WELL-BEING: AN EMPIRICAL EXPLORATION ON HUMAN NEEDS AND HUMAN INTERDEPENDENCY
}

\author{
Aisyah Abu Bakar', Mariana Mohamed Osman², Syahriah Bachok ${ }^{3}$, Mansor \\ Ibrahim $^{4}$ \& Muhammad Faris Abdullah ${ }^{5}$ \\ ${ }^{1,2,3,4,5}$ Kulliyyah of Architecture and Environmental Design \\ INTERNATIONAL ISLAMIC UNIVERSITY MALAYSIA
}

\begin{abstract}
This study is a part of an ongoing research to discover subjective indicators of sustainable well-being for Malaysia. Initial findings recognized two important notions of subjective measures of sustainable well-being. The first notion suggested that sustainable well-being manifested in human interdependency. The second notion suggested that human interdependency is attained when human needs are fulfilled. Maslow's Hierarchy of Needs was adopted to indicate the stages and examples of each needs. There were eight stages of human needs which were adapted into 24 common human needs substituted under eight components of three human needs dimensions. The dimensions were (i) basic necessities, (ii) complimentary needs, and (iii) desired opportunities. It was hypothesized that human needs influence the level of human interdependency. This paper delivers an empirical analysis testing the effects of human needs on human interdependency. The study intends to determine the influence of human needs on human interdependency. Questionnaire survey was conducted and 894 reliable samples were gathered. 192 Independent Sample T-Tests were conducted to determine statistical difference in levels of eight components of human interdependency, between respondents who claimed difficult and respondents who claimed easy to attain 24 human needs in the past year. There were statistically significant differences in most of the components of human interdependency between groups of 24 human needs. The empirical study conducted in the central regions of a developing and multicultural country, Malaysia, is a useful reference to subjective well-being studies piloted in areas of similar characteristics.
\end{abstract}

Keyword: sustainable well-being, human interdependency, human needs 
Aisyah Abu Bakar, Mariana Mohamed Osman, Syahriah Bachok, Mansor Ibrahim \& Muhammad Faris Abdullah Sustainable Well-Being: An Empirical Exploration on Human Needs and Human Interdependency

\section{INTRODUCTION}

Human interdependency (HI) is growingly discussed in the field positive psychology as a strong determinant to sustainable well-being (Kjell, 2011; O'Brien, 2012). HI place attention on the change in one's well-being resulting from one's intentional and unintentional contribution to others well-being. Two recognized dimensions of human interdependency are (i) human interdependence with other humans ( $\mathrm{HIH})$, and (ii) human interdependence with the environment (HIE) (Bakar et al., 2015a, 2015b, 2015c). The concept of HI is similar to selfactualization and self-transcends of Maslow's Hierarchy of Needs (MHON) (Tay \& Diener, 2011; Kjell, 2011).

MHON which often depicted in hierarchical pyramid was first introduced in 1943 to recognize pattern of human motivation in fulfilling human needs. The most urgent needs were placed at the bottom of the pyramid. In order for a person to be motivated in achieving higher needs, the lower needs must be fulfilled (Geller, 2015; Tay \& Diener, 2011). The MHON was adopted in this study to indicate examples of human needs to represent every stage of MHON. 24 common human needs were identified and substituted under eight components of three human needs dimensions (refer to Table 2). Basic necessities represent survival factors that meant without it living system is disrupted. Complimentary needs comprise of needs which may cause difficulties in life if they were not met. Desired opportunities represent human needs that fulfil human potential, although without them lives would not be difficult. It was hypothesized that human needs influence the level of human interdependency (Tay \& Diener, 2011).

\section{METHODOLOGY}

The survey questionnaires were conducted in Selangor, Putrajaya and Kuala Lumpur between November and December 2015. The survey was targeted at working Malaysians age from 18 to 65 years old. 1000 survey questionnaires were distributed and 894 samples were found reliable for statistical tests. The respondents were inquired to state whether fulfilling 24 human needs (refer to Table 1) were 'difficult' or 'easy' for them in the past year (2015). The respondents were also inquired to rate their levels of human interdependency under 80 self-reported statements. 40 of the statements account for $\mathrm{HIH}$ and the remaining 40 statements account for HIE. The components of HIH include (i) personal empowerment, (ii) positive relations, (iii) organizational opportunity, and (iv) community mobilization6,7. The components of HIE include (i) personality and lifestyle, (ii) interaction with nature, (iii) attitude and proenvironmental behaviour, and (iv) external condition to behavior5,7. Every 10 statements represent one component of HIH or HIE. Responses for the selfreported statements were on Likert-type scale that range from $0=$ 'highly false' to $10=$ 'highly true'. The scores were averaged to represent the overall score for each component of HIH and HIE (refer to Table 2). 
PLANNING MALAYSIA

Journal of the Malaysian Institute of Planners (2016)

Table 1 Dimensions and Components of Human Needs - Independent Variables

\begin{tabular}{|c|c|c|c|c|c|}
\hline Dimension & $\begin{array}{l}\text { Components: } \\
\text { MHON }\end{array}$ & Code & Human Needs & $\begin{array}{l}\text { Diff. } \\
(\%)\end{array}$ & $\begin{array}{l}\text { Easy } \\
(\%)\end{array}$ \\
\hline \multirow{10}{*}{$\begin{array}{l}\text { (D1) } \\
\text { Basic Necessities } \\
\text { Without it living } \\
\text { system is disrupted }\end{array}$} & \multirow{4}{*}{$\begin{array}{l}\text { C1: Biological \& } \\
\text { Physiological } \\
\text { Needs } \\
\text { Survival elements }\end{array}$} & HN 1 & Nutritious food & 6.6 & 93.4 \\
\hline & & HN 2 & Medical treatment & 8.6 & 91.4 \\
\hline & & $\mathrm{HN} 3$ & $\begin{array}{l}\text { Clean water for washing and } \\
\text { drinking }\end{array}$ & 5.5 & 94.5 \\
\hline & & $\mathrm{HN} 4$ & Well-functioned toilet & 28.3 & 71.7 \\
\hline & \multirow{6}{*}{$\begin{array}{l}\text { C2: Safety \& } \\
\text { Security Needs } \\
\text { Long-term survival } \\
\text { and stability: }\end{array}$} & HN 5 & Adequate electricity supply & 7.6 & 92.4 \\
\hline & & HN 6 & $\begin{array}{l}\text { Affordable house \& } \\
\text { surrounding amenities }\end{array}$ & 22.3 & 77.7 \\
\hline & & HN 7 & Financial stability & 49.6 & 50.4 \\
\hline & & HN 8 & Personal security & 46.9 & 53.1 \\
\hline & & HN 9 & Clean air & 42.8 & 57.2 \\
\hline & & HN 10 & Health and wellness assurance & 43.6 & 56.4 \\
\hline \multirow{6}{*}{$\begin{array}{l}\text { (D2) } \\
\text { Complimentary } \\
\text { Needs } \\
\text { Without it, living } \\
\text { system is not } \\
\text { disrupted, but } \\
\text { lives would be } \\
\text { difficult }\end{array}$} & \multirow{4}{*}{$\begin{array}{l}\text { C3: Belonging \& } \\
\text { Love Needs } \\
\text { Affiliation and } \\
\text { acceptance }\end{array}$} & HN 11 & $\begin{array}{l}\text { Balance between personal \& } \\
\text { work time }\end{array}$ & 52.6 & 47.4 \\
\hline & & HN 12 & Social Tolerance & 44.7 & 55.3 \\
\hline & & HN 13 & Communication line & 3.9 & 96.1 \\
\hline & & HN 14 & Internet line/ hotspot & 19.2 & 80.8 \\
\hline & \multirow{2}{*}{$\begin{array}{l}\text { C4: Esteem Needs } \\
\text { Achievement and } \\
\text { recognition }\end{array}$} & HN 15 & Primary school education & 3.0 & 97.0 \\
\hline & & HN 16 & Secondary school education & 5.8 & 94.2 \\
\hline \multirow{8}{*}{$\begin{array}{l}\text { (D2) } \\
\text { Desired } \\
\text { Opportunities } \\
\text { Without it, living } \\
\text { system is not } \\
\text { disrupted, and } \\
\text { lives would not be } \\
\text { difficult }\end{array}$} & C5: Cognitive & HN 17 & Tertiary/higher education & 12.1 & 87.9 \\
\hline & $\begin{array}{l}\text { Needs } \\
\text { Knowledge \& } \\
\text { understanding }\end{array}$ & HN 18 & Job opportunity & 41.6 & 58.4 \\
\hline & \multirow{2}{*}{$\begin{array}{l}\text { C6: Aesthetic } \\
\text { Needs } \\
\text { Order and beauty }\end{array}$} & HN 19 & $\begin{array}{l}\text { Clean \& well-maintained } \\
\text { recreational park }\end{array}$ & 42.7 & 57.3 \\
\hline & & HN 20 & $\begin{array}{l}\text { Diversity of plants and animal } \\
\text { species }\end{array}$ & 46.0 & 54.0 \\
\hline & C7: Self- & HN 21 & Rights to choose leaders & 59.3 & 40.7 \\
\hline & $\begin{array}{l}\text { Actualization } \\
\text { Needs } \\
\text { Realization of } \\
\text { potential }\end{array}$ & HN 22 & Freedom of speech & 64.8 & 35.2 \\
\hline & \multirow{2}{*}{$\begin{array}{l}\text { C8: Self- } \\
\text { Transcends Needs } \\
\text { Self-functioning }\end{array}$} & HN 23 & Corruption free opportunities & 69.9 & 30.1 \\
\hline & & HN 24 & $\begin{array}{l}\text { Freedom to express arts \& } \\
\text { diversity }\end{array}$ & 48.8 & 51.2 \\
\hline
\end{tabular}

Table 2 Human Interdependency - Dependent Variables

\begin{tabular}{llll}
\hline Human Interdependency $(\mathrm{HIH})$ & Code & $\begin{array}{l}\text { Components of Human } \\
\text { Interdependency }\end{array}$ & Mean \\
\hline \multirow{2}{*}{$\begin{array}{l}\text { Human Interdependence with other } \\
\text { Humans } \\
(\mathrm{HIH})\end{array}$} & PE & Personal Empowerment \\
\cline { 2 - 3 } & PR & Positive Relations \\
\cline { 2 - 3 } & OO & Organizational Opportunity \\
\cline { 2 - 3 } & CM & Community Mobilization \\
\hline
\end{tabular}


Aisyah Abu Bakar, Mariana Mohamed Osman, Syahriah Bachok, Mansor Ibrahim \& Muhammad Faris Abdullah Sustainable Well-Being: An Empirical Exploration on Human Needs and Human Interdependency

\begin{tabular}{lll}
\hline Human Interdependence with the & IN & Interaction with Nature \\
\cline { 2 - 3 } $\begin{array}{l}\text { Environment } \\
\text { (HIE) }\end{array}$ & AP & $\begin{array}{l}\text { Attitude and Pro-Environmental } \\
\text { Behaviour }\end{array}$ \\
\cline { 2 - 3 } & EC & External Condition to Behaviour \\
\hline
\end{tabular}

Independent sample t-tests were conducted to determine the levels of human interdependency (HI) of four components of $\mathrm{HIH}$ and four components HIE (refer to Table 1) between respondents who claimed 'easy' and respondents who claimed 'difficult' to fulfil 24 human needs (HN). The Independent T-Test assumed that the variance of the two groups being compared to be equal. The homogeneity of variance was tested using Levene's Test for Equality of Variances. It is to be observed that for some of the HNs, the number of respondents who claimed 'difficult' and the number of respondents who claimed 'easy' extremely differ. Thus violate the assumption of homogeneity and the tests can result to Type 1 error. In order to overcome the violation of the homogeneity assumption, the Welch-Satterthwaite method were used to adjust the degree of freedom (df) and resulted to increased p-value above the significance level of 0.05 .

\section{EMPIRICAL FINDINGS}

Descriptive statistics in Table 3 indicated that the mean of all components of $\mathrm{HIH}$ was higher for respondents who claimed easy than those claimed difficult in fulfilling all of the HNs. Almost all mean of HIE components were higher for respondents who claimed easy than those claimed difficult in fulfilling all of the HNs. A few outputs yielded intriguing descriptive statistics for HIE components (HN 1, IN; HN 3, PL; HN 13, IN; HN 15, EC and AP).

Out of 192 statistical analyses conducted, 140 of them yielded significant outputs $(\mathrm{p}<0.05)$. Thus implied that HI significantly differ between groups of HNs. Out of the 140 significant outputs, 65 of the tests were between groups of equal variance $(p>0.05)$ and 75 of the tests were between groups of unequal variance $(p<0.05)$ based on the Levene's Tests of Equality of Variance (refer to Table 3 ). The analyses on HIH components yielded slightly more significant statistical results (72 tests) compared to HIE components (68 tests) (refer to Table $4)$.

$12 \mathrm{HNs}$ out of $24 \mathrm{HNs}$ had statistically significant effects on the mean of all $\mathrm{HIH}$ and HIE components. Majority of the mentioned HNs belonged in D1, which meant without fulfilment of these needs, living system would be disrupted. These HNs were $\mathrm{HN} 4$, well-functioned toilet (C1, D1); HN 7, financial stability (C2, D1); HN 8, personal security (C2, D1); HN 9, clean air (C2, D1); HN 10, health and wellness assurance $(\mathrm{C} 2, \mathrm{D} 1)$; HN 11, balance between personal and work time (C3, D2); HN 12, social Tolerance (C3, D2); HN 16, secondary school education $(\mathrm{C} 4, \mathrm{D} 2) ; \mathrm{HN} 19$, clean and well-maintained recreational park (C6, D3); HN 20, diversity of plants and animal species (C6, D3); HN 22, freedom of 
speech (C7, D3); and finally, $\mathrm{HN} 24$, freedom to express arts and diversity (C8, D3). Among the 12 HNs mentioned, one in particular (HN 16, secondary school education) had equal variance between groups in all of the tests. Overall, the results implied that the mean of HIH and HIE were statistically higher for respondents who claimed that attaining the mentioned human needs were easy compared to those who claimed difficult.

The mean of PE (personal empowerment) in particular significantly differ between groups of 21 HNs. 10 of the statistical tests had groups of equal variance. The $\mathrm{HNs}$ were $\mathrm{HN} 1$, nutritious food $(\mathrm{C} 1, \mathrm{D} 1) ; \mathrm{H} 2$, medical treatment $(\mathrm{C} 1, \mathrm{D} 1)$; HN 3, clean water for washing and drinking (C1, D1); HN 4, wellfunctioned toilet (C1, D1); HN 5, adequate electricity supply (C2, D1); HN 6, affordable houses and surrounding amenities $(\mathrm{C} 2, \mathrm{D} 1)$; HN 14, internet line or hotspot (C3, D2); H16, secondary school education $(\mathrm{C} 4, \mathrm{D} 2)$; HN 17, tertiary or higher education (C5, D3); HN 21, rights to choose leaders (C7, D3). The results implied that the level of personal empowerment was statistically higher for respondents who claimed that attaining the mentioned human needs were easy compared to those who claimed difficult.

The analysis yielded intriguing outputs for $\mathrm{HN} 13$ and HN 15. There were no statistical differences in levels of all HIH and HIE components between groups of HN 13 (communication line) and HN 15 (primary school education). Similarly, HN 14 (internet connection or hotspot) and HN 6 (affordable house and surrounding amenities) also had only one significant statistical effect on PE. D2 represents $\mathrm{HN}$ 13, HN 14 and $\mathrm{HN}$ 15, which meant without these needs, living system would not be disrupted but lives would be difficult. HN 6 belongs in D1 which suggested that without it, living system would be disrupted. The results suggested that the level of $\mathrm{HIH}$ and $\mathrm{HIE}$ was relatively independent from fulfilment of (i) affordable house, (ii) communication line, (iii) internet connection, and (iv) primary school education.

Table 3 Human Needs and Human Interdependency

\begin{tabular}{|c|c|c|c|c|c|c|c|c|c|}
\hline \multicolumn{4}{|c|}{ Descriptive Statistics } & \multicolumn{6}{|c|}{ Inferential Statistics } \\
\hline IV & DV & Diff. & Easy & $\mathrm{F}$ & Sig. & $\mathrm{t}$ & $\mathrm{df}$ & Sig. & MD \\
\hline \multirow{5}{*}{$\begin{array}{l}\mathrm{H} \\
\mathrm{N}\end{array}$} & $\mathrm{PP}$ & 6.83 & 7.22 & 0.030 & 0.86 & -2.389 & 892 & 0.02 & -0.39 \\
\hline & PR & 6.81 & 7.26 & 3.955 & 0.04 & -2.271 & 63.5 & 0.03 & -0.45 \\
\hline & $\mathrm{OO}$ & 6.95 & 7.27 & 4.192 & 0.04 & -1.622 & 63.7 & 0.11 & -0.33 \\
\hline & $\mathrm{CM}$ & 6.38 & 6.85 & 1.870 & 0.17 & -2.729 & 892 & 0.01 & -0.47 \\
\hline & PL & 6.98 & 7.05 & 1.247 & 0.26 & -0.439 & 892 & 0.66 & -0.07 \\
\hline \multirow[t]{4}{*}{1} & IN & 6.72 & 6.68 & 5.736 & 0.02 & 0.166 & 63.1 & 0.87 & 0.04 \\
\hline & AP & 6.33 & 6.56 & 0.737 & 0.39 & -1.131 & 892 & 0.26 & -0.23 \\
\hline & EC & 5.94 & 6.37 & 1.424 & 0.23 & -2.140 & 892 & 0.03 & -0.42 \\
\hline & $\mathrm{PP}$ & 6.83 & 7.23 & 0.319 & 0.57 & -2.760 & 892 & 0.01 & -0.39 \\
\hline \multirow{3}{*}{$\begin{array}{l}\mathrm{H} \\
\mathrm{N}\end{array}$} & PR & 6.79 & 7.28 & 2.703 & 0.10 & -3.284 & 892 & 0.00 & -0.48 \\
\hline & $\mathrm{OO}$ & 6.83 & 7.29 & 2.782 & 0.10 & -3.043 & 892 & 0.00 & -0.46 \\
\hline & $\mathrm{CM}$ & 6.48 & 6.85 & 0.809 & 0.37 & -2.443 & 892 & 0.02 & -0.37 \\
\hline \multirow{3}{*}{2} & PL & 6.76 & 7.07 & 1.663 & 0.20 & -2.125 & 892 & 0.03 & -0.31 \\
\hline & IN & 6.55 & 6.69 & 0.837 & 0.36 & -0.906 & 892 & 0.37 & -0.14 \\
\hline & $\mathrm{AP}$ & 6.21 & 6.58 & 0.596 & 0.44 & -2.064 & 892 & 0.04 & -0.37 \\
\hline
\end{tabular}


Aisyah Abu Bakar, Mariana Mohamed Osman, Syahriah Bachok, Mansor Ibrahim \& Muhammad Faris Abdullah Sustainable Well-Being: An Empirical Exploration on Human Needs and Human Interdependency

\begin{tabular}{|c|c|c|c|c|c|c|c|c|c|}
\hline & $\mathrm{EC}$ & 5.99 & 6.37 & 1.860 & 0.17 & -2.194 & 892 & 0.03 & -0.38 \\
\hline \multirow{5}{*}{$\begin{array}{l}\mathrm{H} \\
\mathrm{N}\end{array}$} & PP & 6.76 & 7.22 & 0.088 & 0.77 & -2.615 & 892 & 0.01 & -0.46 \\
\hline & PR & 6.82 & 7.26 & 7.333 & 0.01 & -1.964 & 51.5 & 0.06 & -0.44 \\
\hline & $\mathrm{OO}$ & 7.12 & 7.26 & 1.592 & 0.21 & -0.758 & 892 & 0.45 & -0.14 \\
\hline & CM & 6.64 & 6.83 & 1.115 & 0.29 & -1.003 & 892 & 0.32 & -0.19 \\
\hline & $\mathrm{PL}$ & 7.16 & 7.04 & 0.007 & 0.93 & 0.671 & 892 & 0.50 & 0.12 \\
\hline \multirow[t]{5}{*}{3} & IN & 6.62 & 6.69 & 3.132 & 0.08 & -0.356 & 892 & 0.72 & -0.07 \\
\hline & AP & 6.42 & 6.56 & 0.592 & 0.44 & -0.600 & 892 & 0.55 & -0.13 \\
\hline & $\mathrm{EC}$ & 6.17 & 6.35 & 2.958 & 0.09 & -0.828 & 892 & 0.41 & -0.18 \\
\hline & PP & 7.03 & 7.26 & 0.325 & 0.57 & -2.491 & 892 & 0.01 & -0.22 \\
\hline & PR & 7.04 & 7.31 & 0.148 & 0.70 & -2.884 & 892 & 0.00 & -0.26 \\
\hline \multirow{3}{*}{$\begin{array}{l}\mathrm{H} \\
\mathrm{N}\end{array}$} & $\mathrm{OO}$ & 7.09 & 7.32 & 0.340 & 0.56 & -2.385 & 892 & 0.02 & -0.22 \\
\hline & $\mathrm{CM}$ & 6.53 & 6.93 & 3.985 & 0.05 & -4.227 & 892 & 0.00 & -0.40 \\
\hline & PL & 6.80 & 7.15 & 12.964 & 0.00 & -4.129 & 536.6 & 0.00 & -0.35 \\
\hline \multirow[t]{5}{*}{4} & IN & 6.46 & 6.77 & 0.055 & 0.82 & -3.207 & 892 & 0.00 & -0.32 \\
\hline & AP & 6.25 & 6.66 & 3.870 & 0.04 & -3.887 & 526.0 & 0.00 & -0.41 \\
\hline & $\mathrm{EC}$ & 5.95 & 6.49 & 4.090 & 0.04 & -5.233 & 512.4 & 0.00 & -0.54 \\
\hline & PP & 6.83 & 7.22 & 1.670 & 0.20 & -2.579 & 892 & 0.01 & $\begin{array}{l}-0.39 \\
\end{array}$ \\
\hline & PR & 6.60 & 7.29 & 0.064 & 0.80 & -4.410 & 892 & 0.00 & -0.68 \\
\hline \multirow{3}{*}{$\begin{array}{l}\mathrm{H} \\
\mathrm{N}\end{array}$} & $\mathrm{OO}$ & 6.81 & 7.29 & 0.252 & 0.62 & -2.993 & 892 & 0.00 & -0.48 \\
\hline & $\mathrm{CM}$ & 6.73 & 6.83 & 0.126 & 0.72 & -0.610 & 892 & 0.54 & -0.10 \\
\hline & $\mathrm{PL}$ & 6.69 & 7.08 & 2.672 & 0.10 & -2.463 & 892 & 0.01 & -0.38 \\
\hline \multirow[t]{5}{*}{5} & IN & 6.39 & 6.71 & 0.047 & 0.83 & -1.865 & 892 & 0.06 & -0.31 \\
\hline & $\mathrm{AP}$ & 6.26 & 6.57 & 1.695 & 0.19 & -1.601 & 892 & 0.11 & -0.31 \\
\hline & $\mathrm{EC}$ & 6.10 & 6.36 & 4.070 & 0.04 & -1.591 & 83.6 & 0.12 & -0.25 \\
\hline & $\mathrm{PP}$ & 7.04 & 7.24 & 0.023 & 0.88 & -2.084 & 892 & 0.04 & -0.20 \\
\hline & PR & 7.11 & 7.27 & 0.826 & 0.36 & -1.596 & 892 & 0.11 & -0.16 \\
\hline \multirow{3}{*}{$\begin{array}{l}\mathrm{H} \\
\mathrm{N}\end{array}$} & $\mathrm{OO}$ & 7.22 & 7.26 & 0.348 & 0.56 & -0.348 & 892 & 0.73 & -0.04 \\
\hline & $\mathrm{CM}$ & 6.72 & 6.85 & 0.269 & 0.60 & -1.211 & 892 & 0.23 & -0.13 \\
\hline & PL & 6.91 & 7.09 & 1.003 & 0.32 & -1.761 & 892 & 0.08 & -0.17 \\
\hline \multirow[t]{5}{*}{6} & IN & 6.72 & 6.67 & 0.004 & 0.95 & 0.505 & 892 & 0.61 & 0.05 \\
\hline & $\mathrm{AP}$ & 6.43 & 6.58 & 4.045 & 0.04 & -1.362 & 354.9 & 0.17 & -0.16 \\
\hline & $\mathrm{EC}$ & 6.28 & 6.35 & 7.279 & 0.01 & -0.702 & 371.5 & 0.48 & -0.08 \\
\hline & $\mathrm{PP}$ & 7.06 & 7.33 & 9.347 & 0.00 & -3.424 & 883.1 & 0.00 & -0.27 \\
\hline & PR & 7.12 & 7.35 & 8.972 & 0.00 & -2.818 & 886.1 & 0.01 & -0.23 \\
\hline \multirow{3}{*}{$\begin{array}{l}\mathrm{H} \\
\mathrm{N}\end{array}$} & $\mathrm{OO}$ & 7.11 & 7.40 & 3.436 & 0.06 & -3.432 & 892 & 0.00 & -0.29 \\
\hline & $\mathrm{CM}$ & 6.61 & 7.02 & 9.828 & 0.00 & -4.799 & 878.8 & 0.00 & -0.41 \\
\hline & PL & 6.89 & 7.21 & 9.380 & 0.00 & -3.890 & 883.8 & 0.00 & -0.32 \\
\hline \multirow[t]{5}{*}{7} & IN & 6.54 & 6.82 & 7.361 & 0.01 & -3.239 & 882.4 & 0.00 & -0.29 \\
\hline & $\mathrm{AP}$ & 6.32 & 6.77 & 6.591 & 0.01 & -4.531 & 880.9 & 0.00 & -0.46 \\
\hline & $\mathrm{EC}$ & 6.02 & 6.65 & 12.635 & 0.00 & -6.662 & 879.6 & 0.00 & -0.64 \\
\hline & PP & 7.00 & 7.36 & 8.750 & 0.00 & -4.500 & 891.7 & 0.00 & -0.36 \\
\hline & PR & 7.10 & 7.36 & 6.277 & 0.01 & -3.166 & 891.5 & 0.00 & -0.26 \\
\hline \multirow{3}{*}{$\begin{array}{l}\mathrm{H} \\
\mathrm{N}\end{array}$} & $\mathrm{OO}$ & 7.12 & 7.37 & 1.903 & 0.17 & -3.049 & 892 & 0.00 & -0.26 \\
\hline & $\mathrm{CM}$ & 6.64 & 6.98 & 9.011 & 0.00 & -3.952 & 891.9 & 0.00 & -0.34 \\
\hline & PL & 6.83 & 7.24 & 6.704 & 0.01 & -5.131 & 891.0 & 0.00 & -0.42 \\
\hline \multirow[t]{5}{*}{8} & IN & 6.50 & 6.84 & 4.397 & 0.04 & -3.874 & 891.7 & 0.00 & -0.34 \\
\hline & AP & 6.31 & 6.75 & 3.968 & 0.04 & -4.383 & 891.9 & 0.00 & -0.44 \\
\hline & $\mathrm{EC}$ & 6.07 & 6.57 & 15.110 & 0.00 & -5.186 & 889.2 & 0.00 & -0.50 \\
\hline & $\mathrm{PP}$ & 7.10 & 7.26 & 4.859 & 0.03 & -2.022 & 857.1 & 0.04 & -0.16 \\
\hline & PR & 7.11 & 7.33 & 8.984 & 0.00 & -2.711 & 869.1 & 0.01 & -0.22 \\
\hline \multirow{3}{*}{$\begin{array}{l}\mathrm{H} \\
\mathrm{N}\end{array}$} & $\mathrm{OO}$ & 7.10 & 7.37 & 4.154 & 0.04 & -3.253 & 856.8 & 0.00 & -0.27 \\
\hline & $\mathrm{CM}$ & 6.61 & 6.97 & 6.748 & 0.01 & -4.259 & 869.0 & 0.00 & -0.36 \\
\hline & PL & 6.93 & 7.14 & 4.636 & 0.03 & -2.464 & 845.8 & 0.01 & -0.20 \\
\hline \multirow[t]{3}{*}{9} & IN & 6.56 & 6.78 & 1.865 & 0.17 & -2.436 & 892 & 0.02 & -0.22 \\
\hline & AP & 6.34 & 6.70 & 8.790 & 0.00 & -3.603 & 882.1 & 0.00 & -0.36 \\
\hline & $\mathrm{EC}$ & 6.10 & 6.52 & 0.716 & 0.40 & -4.318 & 892 & 0.00 & -0.42 \\
\hline
\end{tabular}


PLANNING MALAYSIA

Journal of the Malaysian Institute of Planners (2016)

\begin{tabular}{|c|c|c|c|c|c|c|c|c|c|}
\hline \multirow{4}{*}{$\begin{array}{l}\mathrm{H} \\
\mathrm{N}\end{array}$} & PP & 7.04 & 7.31 & 11.697 & 0.00 & -3.330 & 880.8 & 0.00 & -0.26 \\
\hline & PR & 7.08 & 7.35 & 13.156 & 0.00 & -3.394 & 884.7 & 0.00 & -0.28 \\
\hline & $\mathrm{OO}$ & 7.04 & 7.42 & 8.981 & 0.00 & -4.514 & 867.3 & 0.00 & -0.38 \\
\hline & $\mathrm{CM}$ & 6.58 & 7.01 & 21.280 & 0.00 & -5.170 & 889.7 & 0.00 & -0.43 \\
\hline \multirow{4}{*}{10} & PL & 6.76 & 7.27 & 15.788 & 0.00 & -6.364 & 883.1 & 0.00 & -0.51 \\
\hline & IN & 6.53 & 6.80 & 7.946 & 0.01 & -3.071 & 879.6 & 0.00 & -0.27 \\
\hline & AP & 6.28 & 6.75 & 7.156 & 0.01 & -4.665 & 873.9 & 0.00 & -0.47 \\
\hline & $\mathrm{EC}$ & 6.00 & 6.60 & 5.128 & 0.02 & -6.342 & 867.0 & 0.00 & -0.61 \\
\hline \multirow{5}{*}{$\begin{array}{l}\mathrm{H} \\
\mathrm{N}\end{array}$} & $\mathrm{PP}$ & 7.08 & 7.33 & 6.566 & 0.01 & -3.101 & 862.6 & 0.00 & -0.25 \\
\hline & PR & 7.13 & 7.34 & 4.938 & 0.03 & -2.528 & 871.8 & 0.01 & -0.21 \\
\hline & $\mathrm{OO}$ & 7.11 & 7.41 & 5.203 & 0.02 & -3.557 & 871.6 & 0.00 & -0.30 \\
\hline & $\mathrm{CM}$ & 6.65 & 7.01 & 10.794 & 0.00 & -4.211 & 856.0 & 0.00 & -0.36 \\
\hline & PL & 6.86 & 7.26 & 8.756 & 0.00 & -4.935 & 851.3 & 0.00 & -0.41 \\
\hline \multirow[t]{5}{*}{11} & IN & 6.54 & 6.84 & 1.286 & 0.26 & -3.389 & 892 & 0.00 & -0.30 \\
\hline & AP & 6.26 & 6.87 & 0.626 & 0.43 & -6.113 & 892 & 0.00 & -0.61 \\
\hline & $\mathrm{EC}$ & 6.02 & 6.69 & 0.342 & 0.56 & -6.895 & 892 & 0.00 & -0.66 \\
\hline & PP & 7.07 & 7.29 & 9.487 & 0.00 & -2.810 & 887.5 & 0.01 & -0.22 \\
\hline & PR & 7.12 & 7.33 & 9.411 & 0.00 & -2.501 & 883.0 & 0.01 & -0.20 \\
\hline \multirow{3}{*}{$\begin{array}{l}\mathrm{H} \\
\mathrm{N}\end{array}$} & $\mathrm{OO}$ & 7.12 & 7.36 & 10.817 & 0.00 & -2.916 & 882.1 & 0.00 & -0.24 \\
\hline & CM & 6.60 & 6.99 & 13.523 & 0.00 & -4.605 & 888.5 & 0.00 & -0.39 \\
\hline & PL & 6.81 & 7.24 & 20.994 & 0.00 & -5.287 & 891.2 & 0.00 & -0.43 \\
\hline \multirow[t]{5}{*}{12} & IN & 6.59 & 6.76 & 9.432 & 0.00 & -1.991 & 883.3 & 0.04 & -0.18 \\
\hline & AP & 6.30 & 6.75 & 4.017 & 0.04 & -4.433 & 880.0 & 0.00 & -0.45 \\
\hline & $\mathrm{EC}$ & 6.02 & 6.59 & 1.282 & 0.26 & -5.893 & 892 & 0.00 & -0.57 \\
\hline & PP & 6.99 & 7.20 & 0.018 & 0.89 & -1.026 & 892 & 0.31 & -0.21 \\
\hline & PR & 6.93 & 7.25 & 0.843 & 0.36 & -1.475 & 892 & 0.14 & -0.31 \\
\hline \multirow{3}{*}{$\begin{array}{l}\mathrm{H} \\
\mathrm{N}\end{array}$} & $\mathrm{OO}$ & 7.14 & 7.26 & 0.179 & 0.67 & -0.518 & 892 & 0.60 & -0.11 \\
\hline & $\mathrm{CM}$ & 6.55 & 6.83 & 0.092 & 0.76 & -1.278 & 892 & 0.20 & -0.28 \\
\hline & PL & 6.91 & 7.05 & 0.343 & 0.56 & -0.666 & 892 & 0.51 & -0.14 \\
\hline \multirow[t]{5}{*}{13} & IN & 6.72 & 6.68 & 0.329 & 0.57 & 0.157 & 892 & 0.88 & 0.04 \\
\hline & $\mathrm{AP}$ & 6.22 & 6.56 & 0.509 & 0.48 & -1.297 & 892 & 0.20 & -0.34 \\
\hline & $\mathrm{EC}$ & 6.29 & 6.34 & 0.271 & 0.60 & -0.192 & 892 & 0.85 & -0.05 \\
\hline & PP & 6.83 & 7.28 & 1.015 & 0.31 & -4.510 & 892 & 0.00 & -0.46 \\
\hline & PR & 7.22 & 7.24 & 4.407 & 0.04 & -0.181 & 240.9 & 0.86 & -0.02 \\
\hline \multirow{3}{*}{$\begin{array}{l}\mathrm{H} \\
\mathrm{N}\end{array}$} & $\mathrm{OO}$ & 7.11 & 7.29 & 3.754 & 0.05 & -1.682 & 892 & 0.09 & -0.18 \\
\hline & $\mathrm{CM}$ & 6.71 & 6.84 & 0.474 & 0.49 & -1.193 & 892 & 0.23 & -0.13 \\
\hline & PL & 6.84 & 7.10 & 12.024 & 0.00 & -2.255 & 234.1 & 0.03 & -0.26 \\
\hline \multirow[t]{5}{*}{14} & IN & 6.56 & 6.71 & 0.439 & 0.51 & -1.290 & 892 & 0.20 & -0.15 \\
\hline & $\mathrm{AP}$ & 6.51 & 6.56 & 0.619 & 0.43 & -0.339 & 892 & 0.74 & -0.04 \\
\hline & $\mathrm{EC}$ & 6.26 & 6.36 & 1.343 & 0.25 & -0.772 & 892 & 0.44 & -0.10 \\
\hline & $\mathrm{PP}$ & 6.90 & 7.20 & 0.059 & 0.81 & -1.285 & 892 & 0.20 & -0.30 \\
\hline & PR & 6.84 & 7.25 & 0.024 & 0.88 & -1.677 & 892 & 0.09 & -0.40 \\
\hline \multirow{3}{*}{$\begin{array}{l}\mathrm{H} \\
\mathrm{N}\end{array}$} & $\mathrm{OO}$ & 6.94 & 7.26 & 0.410 & 0.52 & -1.280 & 892 & 0.20 & -0.32 \\
\hline & $\mathrm{CM}$ & 6.75 & 6.82 & 1.425 & 0.23 & -0.271 & 892 & 0.79 & -0.07 \\
\hline & PL & 6.96 & 7.05 & 1.942 & 0.16 & -0.363 & 892 & 0.72 & -0.09 \\
\hline \multirow[t]{5}{*}{15} & IN & 6.57 & 6.69 & 1.881 & 0.17 & -0.457 & 892 & 0.65 & -0.12 \\
\hline & $\mathrm{AP}$ & 6.69 & 6.54 & 0.538 & 0.46 & 0.501 & 892 & 0.62 & 0.15 \\
\hline & $\mathrm{EC}$ & 6.57 & 6.33 & 1.003 & 0.32 & 0.849 & 892 & 0.40 & 0.24 \\
\hline & $\mathrm{PP}$ & 6.58 & 7.23 & 0.007 & 0.93 & -3.794 & 892 & 0.00 & -0.65 \\
\hline & PR & 6.54 & 7.28 & 0.189 & 0.66 & -4.193 & 892 & 0.00 & -0.73 \\
\hline \multirow{3}{*}{$\begin{array}{l}\mathrm{H} \\
\mathrm{N}\end{array}$} & $\mathrm{OO}$ & 6.39 & 7.31 & 0.946 & 0.33 & -5.124 & 892 & 0.00 & -0.91 \\
\hline & $\mathrm{CM}$ & 6.37 & 6.85 & 0.060 & 0.81 & -2.623 & 892 & 0.01 & -0.48 \\
\hline & PL & 6.40 & 7.09 & 1.932 & 0.17 & -3.902 & 892 & 0.00 & -0.68 \\
\hline \multirow[t]{3}{*}{16} & IN & 6.10 & 6.72 & 0.112 & 0.74 & -3.249 & 892 & 0.00 & -0.62 \\
\hline & AP & 6.13 & 6.57 & 0.054 & 0.82 & -2.024 & 892 & 0.04 & -0.44 \\
\hline & $\mathrm{EC}$ & 6.02 & 6.36 & 3.056 & 0.08 & -1.636 & 892 & 0.10 & -0.34 \\
\hline $\mathrm{H}$ & PP & 6.76 & 7.25 & 0.036 & 0.85 & -4.010 & 892 & 0.00 & -0.49 \\
\hline
\end{tabular}


Aisyah Abu Bakar, Mariana Mohamed Osman, Syahriah Bachok, Mansor Ibrahim \& Muhammad Faris Abdullah Sustainable Well-Being: An Empirical Exploration on Human Needs and Human Interdependency

\begin{tabular}{|c|c|c|c|c|c|c|c|c|c|}
\hline \multirow[t]{2}{*}{$\mathrm{N}$} & PR & 6.67 & 7.31 & 0.025 & 0.88 & -5.162 & 892 & 0.00 & -0.65 \\
\hline & $\mathrm{OO}$ & 6.70 & 7.33 & 0.973 & 0.32 & -4.898 & 892 & 0.00 & -0.63 \\
\hline \multirow[t]{7}{*}{17} & $\mathrm{CM}$ & 6.43 & 6.87 & 0.946 & 0.33 & -3.374 & 892 & 0.00 & -0.44 \\
\hline & PL & 6.72 & 7.09 & 0.042 & 0.84 & -2.983 & 892 & 0.00 & -0.38 \\
\hline & IN & 6.41 & 6.72 & 0.121 & 0.73 & -2.297 & 892 & 0.02 & -0.31 \\
\hline & $\mathrm{AP}$ & 6.40 & 6.57 & 2.132 & 0.15 & -1.046 & 892 & 0.30 & -0.16 \\
\hline & $\mathrm{EC}$ & 5.97 & 6.39 & 8.157 & 0.00 & -3.096 & 147.4 & 0.00 & -0.42 \\
\hline & $\mathrm{PP}$ & 7.12 & 7.25 & 1.423 & 0.23 & -1.635 & 892 & 0.10 & -0.13 \\
\hline & PR & 7.14 & 7.30 & 0.001 & 0.98 & -1.917 & 892 & 0.06 & -0.16 \\
\hline \multirow{3}{*}{$\begin{array}{l}\mathrm{H} \\
\mathrm{N}\end{array}$} & $\mathrm{OO}$ & 7.11 & 7.35 & 2.769 & 0.10 & -2.874 & 892 & 0.00 & -0.25 \\
\hline & $\mathrm{CM}$ & 6.67 & 6.92 & 3.705 & 0.06 & -2.901 & 892 & 0.00 & -0.25 \\
\hline & PL & 6.85 & 7.19 & 5.621 & 0.02 & -4.054 & 839.2 & 0.00 & -0.33 \\
\hline \multirow[t]{5}{*}{18} & IN & 6.59 & 6.75 & 1.041 & 0.31 & -1.821 & 892 & 0.07 & -0.16 \\
\hline & $\mathrm{AP}$ & 6.40 & 6.65 & 2.947 & 0.09 & -2.494 & 892 & 0.01 & -0.26 \\
\hline & EC & 6.05 & 6.55 & 5.666 & 0.02 & -5.171 & 830.6 & 0.00 & -0.50 \\
\hline & $\mathrm{PP}$ & 7.04 & 7.31 & 4.560 & 0.03 & -3.445 & 852.2 & 0.00 & -0.28 \\
\hline & PR & 7.01 & 7.40 & 4.740 & 0.03 & -4.669 & 844.4 & 0.00 & -0.38 \\
\hline \multirow{3}{*}{$\begin{array}{l}\mathrm{H} \\
\mathrm{N}\end{array}$} & $\mathrm{OO}$ & 7.05 & 7.41 & 1.750 & 0.19 & -4.238 & 892 & 0.00 & -0.36 \\
\hline & $\mathrm{CM}$ & 6.58 & 7.00 & 6.378 & 0.01 & -4.887 & 858.4 & 0.00 & -0.41 \\
\hline & PL & 6.88 & 7.18 & 19.493 & 0.00 & -3.699 & 883.4 & 0.00 & -0.30 \\
\hline \multirow[t]{5}{*}{19} & IN & 6.50 & 6.82 & 4.231 & 0.04 & -3.620 & 862.0 & 0.00 & -0.32 \\
\hline & $\mathrm{AP}$ & 6.23 & 6.78 & 0.250 & 0.62 & -5.419 & 892 & 0.00 & -0.55 \\
\hline & EC & 5.89 & 6.68 & 3.949 & 0.04 & -8.321 & 844.1 & 0.00 & -0.79 \\
\hline & PP & 7.09 & 7.28 & 4.878 & 0.03 & -2.412 & 884.6 & 0.02 & -0.19 \\
\hline & PR & 7.04 & 7.40 & 6.570 & 0.01 & -4.443 & 886.4 & 0.00 & -0.36 \\
\hline \multirow{3}{*}{$\begin{array}{l}\mathrm{H} \\
\mathrm{N}\end{array}$} & $\mathrm{OO}$ & 7.08 & 7.40 & 1.480 & 0.22 & -3.689 & 892 & 0.00 & -0.31 \\
\hline & $\mathrm{CM}$ & 6.57 & 7.03 & 4.943 & 0.03 & -5.430 & 877.6 & 0.00 & -0.46 \\
\hline & PL & 6.91 & 7.17 & 14.561 & 0.00 & -3.236 & 891.7 & 0.00 & -0.26 \\
\hline \multirow[t]{5}{*}{20} & IN & 6.48 & 6.86 & 1.073 & 0.30 & -4.268 & 892 & 0.00 & -0.38 \\
\hline & AP & 6.30 & 6.76 & 1.329 & 0.25 & -4.462 & 892 & 0.00 & -0.45 \\
\hline & $\mathrm{EC}$ & 5.93 & 6.69 & 4.667 & 0.03 & -8.070 & 883.4 & 0.00 & -0.76 \\
\hline & $\mathrm{PP}$ & 7.12 & 7.30 & 8.060 & 0.01 & -2.097 & 734.9 & 0.04 & -0.17 \\
\hline & PR & 7.19 & 7.30 & 2.091 & 0.15 & -1.389 & 892 & 0.17 & -0.12 \\
\hline \multirow{3}{*}{$\begin{array}{l}\mathrm{H} \\
\mathrm{N}\end{array}$} & $\mathrm{OO}$ & 7.20 & 7.33 & 4.430 & 0.04 & -1.486 & 743.0 & 0.14 & -0.13 \\
\hline & $\mathrm{CM}$ & 6.70 & 6.99 & 3.093 & 0.08 & -3.267 & 892 & 0.00 & -0.28 \\
\hline & PL & 6.91 & 7.25 & 3.017 & 0.08 & -4.118 & 892 & 0.00 & -0.34 \\
\hline \multirow[t]{5}{*}{21} & IN & 6.60 & 6.80 & 1.449 & 0.23 & -2.203 & 892 & 0.03 & -0.20 \\
\hline & AP & 6.40 & 6.76 & 6.011 & 0.01 & -3.375 & 725.5 & 0.00 & -0.36 \\
\hline & $\mathrm{EC}$ & 6.12 & 6.66 & 0.113 & 0.74 & -5.474 & 892 & 0.00 & -0.54 \\
\hline & $\mathrm{PP}$ & 7.06 & 7.43 & 9.705 & 0.00 & -4.281 & 596.1 & 0.00 & -0.37 \\
\hline & PR & 7.15 & 7.39 & 5.567 & 0.02 & -2.707 & 606.3 & 0.01 & -0.24 \\
\hline \multirow{3}{*}{$\begin{array}{l}\mathrm{H} \\
\mathrm{N}\end{array}$} & $\mathrm{OO}$ & 7.15 & 7.44 & 5.340 & 0.02 & -3.152 & 607.2 & 0.00 & -0.28 \\
\hline & $\mathrm{CM}$ & 6.71 & 7.02 & 4.337 & 0.04 & -3.418 & 597.1 & 0.00 & -0.31 \\
\hline & PL & 6.88 & 7.35 & 8.468 & 0.00 & -5.337 & 579.8 & 0.00 & -0.47 \\
\hline \multirow[t]{5}{*}{22} & IN & 6.56 & 6.91 & 4.448 & 0.04 & -3.753 & 596.7 & 0.00 & -0.36 \\
\hline & AP & 6.39 & 6.84 & 6.582 & 0.01 & -4.083 & 586.7 & 0.00 & -0.45 \\
\hline & $\mathrm{EC}$ & 6.09 & 6.80 & 3.791 & 0.05 & -7.186 & 892 & 0.00 & -0.72 \\
\hline & $\mathrm{PP}$ & 7.12 & 7.37 & 5.828 & 0.02 & -2.764 & 476.4 & 0.01 & -0.25 \\
\hline & PR & 7.20 & 7.31 & 3.730 & 0.05 & -1.255 & 892 & 0.21 & -0.11 \\
\hline \multirow{6}{*}{$\begin{array}{l}\mathrm{H} \\
\mathrm{N} \\
23\end{array}$} & $\mathrm{OO}$ & 7.22 & 7.33 & 6.034 & 0.01 & -1.211 & 473.3 & 0.23 & -0.12 \\
\hline & $\mathrm{CM}$ & 6.76 & 6.95 & 1.876 & 0.17 & -2.056 & 892 & 0.04 & -0.19 \\
\hline & PL & 6.98 & 7.21 & 8.905 & 0.00 & -2.490 & 452.1 & 0.01 & -0.24 \\
\hline & IN & 6.61 & 6.85 & 1.549 & 0.21 & -2.445 & 892 & 0.02 & -0.24 \\
\hline & AP & 6.46 & 6.76 & 8.832 & 0.00 & -2.553 & 448.3 & 0.01 & -0.30 \\
\hline & EC & 6.20 & 6.67 & 1.381 & 0.24 & -4.476 & 892 & 0.00 & -0.47 \\
\hline \multirow{3}{*}{$\begin{array}{l}\mathrm{H} \\
\mathrm{N}\end{array}$} & $\mathrm{PP}$ & 7.08 & 7.30 & 9.516 & 0.00 & -2.738 & 888.6 & 0.01 & -0.22 \\
\hline & PR & 7.13 & 7.33 & 17.587 & 0.00 & -2.353 & 882.5 & 0.02 & -0.19 \\
\hline & $\mathrm{OO}$ & 7.10 & 7.39 & 13.925 & 0.00 & -3.482 & 888.9 & 0.00 & -0.29 \\
\hline
\end{tabular}


PLANNING MALAYSIA

Journal of the Malaysian Institute of Planners (2016)

\begin{tabular}{|c|c|c|c|c|c|c|c|c|c|}
\hline \multirow[t]{5}{*}{24} & $\mathrm{CM}$ & 6.66 & 6.97 & 12.100 & 0.00 & -3.572 & 887.3 & 0.00 & -0.30 \\
\hline & PL & 6.85 & 7.24 & 16.449 & 0.00 & -4.776 & 878.7 & 0.00 & -0.39 \\
\hline & IN & 6.49 & 6.86 & 4.676 & 0.03 & -4.169 & 890.9 & 0.00 & -0.37 \\
\hline & $\mathrm{AP}$ & 6.31 & 6.78 & 5.863 & 0.02 & -4.705 & 889.6 & 0.00 & -0.47 \\
\hline & $\mathrm{EC}$ & 6.00 & 6.66 & 3.064 & 0.08 & -6.844 & 891.9 & 0.00 & -0.65 \\
\hline
\end{tabular}

Table 3 Summary of Statistical Outputs

\begin{tabular}{|c|c|c|c|c|c|c|c|c|c|c|}
\hline \multirow{2}{*}{\multicolumn{2}{|c|}{ Human Needs }} & \multicolumn{4}{|c|}{$\mathrm{HIH}$} & \multicolumn{4}{|c|}{$\mathrm{HIE}$} & \multirow{2}{*}{ Total Count } \\
\hline & & $\mathrm{PE}$ & PR & $\mathrm{OO}$ & $\mathrm{CM}$ & PL & IN & AP & $\mathrm{EC}$ & \\
\hline \multirow{10}{*}{ D1 } & HN 1 & $* S$ & $\mathrm{~S}$ & $N S$ & $* S$ & $N S$ & $N S$ & $N S$ & $* S$ & $3 * \mathrm{~S} / 1 \mathrm{~S} / 4 N S$ \\
\hline & HN 2 & $* S$ & $* \mathrm{~S}$ & $* S$ & $* S$ & $* \mathrm{~S}$ & $N S$ & $* \mathrm{~S}$ & $* S$ & $7 * \mathrm{~S} / 0 \mathrm{~S} / 1 N S$ \\
\hline & HN 3 & $* S$ & $S$ & $N S$ & $N S$ & $N S$ & $N S$ & $N S$ & $N S$ & $1 * \mathrm{~S} / 1 \mathrm{~S} / 6 N S$ \\
\hline & HN 4 & $* S$ & $* \mathrm{~S}$ & $* S$ & $* \mathrm{~S}$ & $\mathrm{~S}$ & $* \mathrm{~S}$ & $\mathrm{~S}$ & $\mathrm{~S}$ & $5 * \mathrm{~S} / 3 \mathrm{~S} / 0 N S$ \\
\hline & HN 5 & $* \mathrm{~S}$ & $* \mathrm{~S}$ & $* \mathrm{~S}$ & $N S$ & $* \mathrm{~S}$ & $N S$ & $N S$ & $N S$ & $4 * \mathrm{~S} / 0 \mathrm{~S} / 4 N S$ \\
\hline & HN 6 & $* S$ & $N S$ & $N S$ & $N S$ & $N S$ & $N S$ & $N S$ & $N S$ & $1 * \mathrm{~S} / 0 \mathrm{~S} / 7 N S$ \\
\hline & HN 7 & $\mathrm{~S}$ & $\mathrm{~S}$ & $* \mathrm{~S}$ & $\mathrm{~S}$ & $\mathrm{~S}$ & $\mathrm{~S}$ & $\mathrm{~S}$ & $S$ & $1 * \mathrm{~S} / 7 \mathrm{~S} / 0 N S$ \\
\hline & HN 8 & $\mathrm{~S}$ & $\mathrm{~S}$ & $* \mathrm{~S}$ & $\mathrm{~S}$ & $\mathrm{~S}$ & $\mathrm{~S}$ & $\mathrm{~S}$ & $S$ & $1 * \mathrm{~S} / 7 \mathrm{~S} / 0 N S$ \\
\hline & HN 9 & $S$ & $S$ & $\mathrm{~S}$ & $\mathrm{~S}$ & $S$ & $* S$ & $S$ & $S$ & $1 * \mathrm{~S} / 7 \mathrm{~S} / 0 N S$ \\
\hline & HN 10 & $S$ & $S$ & $S$ & $\mathrm{~S}$ & $S$ & $S$ & $S$ & $S$ & $0 * \mathrm{~S} / 8 \mathrm{~S} / 0 N S$ \\
\hline \multirow{6}{*}{$D 2$} & HN 11 & $S$ & $S$ & $S$ & $\mathrm{~S}$ & $S$ & $* \mathrm{~S}$ & $* S$ & $* S$ & $3 * \mathrm{~S} / 5 \mathrm{~S} / 0 N S$ \\
\hline & HN 12 & $S$ & $S$ & $S$ & $\mathrm{~S}$ & $\mathrm{~S}$ & $\mathrm{~S}$ & $\mathrm{~S}$ & $* S$ & $1 * \mathrm{~S} / 7 \mathrm{~S} / 0 N S$ \\
\hline & HN 13 & $N S$ & $N S$ & $N S$ & $N S$ & $N S$ & $N S$ & $N S$ & $N S$ & $0 * \mathrm{~S} / 0 \mathrm{~S} / 8 N S$ \\
\hline & HN 14 & $* S$ & $N S$ & $N S$ & $N S$ & $N S$ & $N S$ & $N S$ & $N S$ & $1 * \mathrm{~S} / 0 \mathrm{~S} / 7 N S$ \\
\hline & HN 15 & $N S$ & $N S$ & $N S$ & $N S$ & $N S$ & $N S$ & $N S$ & $N S$ & $0 * \mathrm{~S} / 0 \mathrm{~S} / 8 N S$ \\
\hline & HN 16 & $* S$ & $* \mathrm{~S}$ & $* \mathrm{~S}$ & $* \mathrm{~S}$ & $* S$ & $* S$ & $* S$ & $* S$ & $8 * S / 0 S / 0 N S$ \\
\hline \multirow{8}{*}{ D3 } & HN 17 & $* S$ & $* \mathrm{~S}$ & $* \mathrm{~S}$ & $* S$ & $* S$ & $* S$ & $N S$ & $\mathrm{~S}$ & $6 * \mathrm{~S} / 1 \mathrm{~S} / 1 N S$ \\
\hline & HN 18 & $N S$ & $N S$ & $* \mathrm{~S}$ & $* S$ & $S$ & $* S$ & $* \mathrm{~S}$ & $S$ & $4 * \mathrm{~S} / 2 \mathrm{~S} / 2 N S$ \\
\hline & HN 19 & $S$ & $S$ & $* \mathrm{~S}$ & $S$ & $S$ & $S$ & $* S$ & $* S$ & $3 * \mathrm{~S} / 5 \mathrm{~S} / 0 N S$ \\
\hline & HN 20 & $\mathrm{~S}$ & $\mathrm{~S}$ & $* \mathrm{~S}$ & $\mathrm{~S}$ & $\mathrm{~S}$ & $* S$ & $* S$ & $S$ & $3 * \mathrm{~S} / 5 \mathrm{~S} / 0 N S$ \\
\hline & HN 21 & $* S$ & $N S$ & $N S$ & $* S$ & $* S$ & $* S$ & $* S$ & ${ }^{*} \mathrm{~S}$ & $6^{*} \mathrm{~S} / 0 \mathrm{~S} / 2 N S$ \\
\hline & HN 22 & $S$ & $S$ & $* \mathrm{~S}$ & $\mathrm{~S}$ & $\mathrm{~S}$ & $S$ & $\mathrm{~S}$ & $* S$ & $2 * \mathrm{~S} / 6 \mathrm{~S} / 0 N S$ \\
\hline & HN 23 & $S$ & $N S$ & $N S$ & $* \mathrm{~S}$ & $S$ & $* S$ & $\mathrm{~S}$ & $* S$ & $3 * \mathrm{~S} / 3 \mathrm{~S} / 2 N S$ \\
\hline & HN 24 & $S$ & $S$ & $\mathrm{~S}$ & $\mathrm{~S}$ & $\mathrm{~S}$ & $\mathrm{~S}$ & $\mathrm{~S}$ & $* S$ & $1 * \mathrm{~S} / 7 \mathrm{~S} / 0 N S$ \\
\hline \multirow{3}{*}{\multicolumn{2}{|c|}{$\begin{array}{l}\text { Total } \\
\text { Count }\end{array}$}} & $10 * \mathrm{~S}$ & $5 * S$ & $11 * \mathrm{~S}$ & $8 * \mathrm{~S}$ & $5 * S$ & $9 * \mathrm{~S}$ & $7 * S$ & $10 * \mathrm{~S}$ & $65 * \mathrm{~S}$ \\
\hline & & $11 \mathrm{~S}$ & $12 \mathrm{~S}$ & $5 \mathrm{~S}$ & $10 \mathrm{~S}$ & $13 \mathrm{~S}$ & $7 \mathrm{~S}$ & $9 \mathrm{~S}$ & $8 \mathrm{~S}$ & $75 \mathrm{~S}$ \\
\hline & & $3 N S$ & $7 N S$ & $8 N S$ & $6 N S$ & $6 N S$ & $8 N S$ & $8 N S$ & $6 N S$ & $52 N S$ \\
\hline \multicolumn{11}{|l|}{$N S$} \\
\hline$\underset{* S}{S}$ & & signif & at bet & en gro & os of $\mathrm{e}$ & vari & e not & umed & & \\
\hline$* \mathrm{~S}$ & : Statistic & signif & at bet & gro & os of e & vari & assu & & & \\
\hline
\end{tabular}

\section{SUMMARY AND CONCLUSION}

There were three general yet inquisitive findings recognized from the statistical results. The following points summarized these findings.

1. The descriptive statistics suggested that fulfilments of HN 1 (nutritious food), HN 3 (clean water for washing and drinking), $\mathrm{HN} 13$ (communication line) and HN 15 (primary school education) were likely lowering the levels of HIE. Yet the mean differences were trivial and the analyses did not yield significant statistical results.

2. In comparison to HIE, HIH was slightly more dependent on $\mathrm{HN}$, particularly PE (personal empowerment). This could be resulting from the fact that the concept of PE is more related to self-actualization in 
Aisyah Abu Bakar, Mariana Mohamed Osman, Syahriah Bachok, Mansor Ibrahim \& Muhammad Faris Abdullah Sustainable Well-Being: An Empirical Exploration on Human Needs and Human Interdependency

MHON, which is closer to the rest of the needs compared self-transcend (refer to Table 2).

3. HIH and HIE were almost independent from HN 6 (affordable housing and surrounding amenities), HN 13 (communication line), HN 14 (internet connection or hotspot) and HN 15 (primary school education). That is, neither difficulties nor ease in fulfilling the mentioned HNs were strongly influencing levels of HIH and HIE.

The future direction of the research is to empirically model HIH and HIE with items representing subjective well-being (SWB) using Structural Equation Modelling. The research will also explore the variance of HIH and HIE between groups of HNs through more robust statistical model.

\section{ACKNOWLEDGEMENT}

This research was supported by MyGRANTS.

\section{REFERENCES}

Bakar, A. A., Osman, M. M., Bachok, S. \& Ibrahim, M. (2015a). An Exploratory Review: Human Interdependence with Environment. In Asia Pacific International Conference on Environment-Behaviour Studies.

Bakar, A. A., Osman, M. M., Bachok, S. \& Abdullah, A. (2015b). An Exploratory Review: Human Interdependence with Environment. In Asia Pacific International Conference on Environment-Behaviour Studies.

Bakar, A. A., Osman, M. M., Bachok, S. \& Ibrahim, M. (2015c). Sustainable Well-being Subjective Indicators: Human Interdependencies with other Humans and with the Environment. In 6th International Conference on Sustainable Future for Human Security.

Geller, E. S. (2015). Why we need Humanistic Behaviorism. ISHN, 49(10), 52.

Kjell, O. N. (2011). Sustainable well-being: A potential synergy between sustainability and well-being research. Review of General Psychology, 15(3), 255-266.

O'Brien, C. (2012). Sustainable happiness and well-being: Future directions for positive psychology. Psychology, 3(12), 1196-1201.

Tay, L., \& Diener, E. (2011). Needs and subjective well-being around the world. Journal of personality and social psychology, 101(2), 354-365. 